\title{
Photodetection of propagating quantum microwaves in circuit QED
}

\author{
Guillermo Romero ${ }^{1}$, Juan José García-Ripoll ${ }^{2}$, \\ and Enrique Solano ${ }^{3,4}$ \\ ${ }^{1}$ Departamento de Física, Universidad de Santiago de Chile, USACH, Casilla 307, \\ Santiago 2, Chile \\ ${ }^{2}$ Instituto de Física Fundamental, CSIC, Serrano 113-bis, 28006 Madrid, Spain \\ ${ }^{3}$ Departamento de Química Física, Universidad del País Vasco - Euskal Herriko \\ Unibertsitatea, Apdo. 644, 48080 Bilbao, Spain \\ ${ }^{4}$ IKERBASQUE, Basque Foundation for Science, Alameda Urquijo 36, 48011 Bilbao, \\ Spain \\ E-mail: enrique_solano@ehu.es
}

\begin{abstract}
We develop the theory of a metamaterial composed of an array of discrete quantum absorbers inside a one-dimensional waveguide that implements a high-efficiency microwave photon detector. A basic design consists of a few metastable superconducting nanocircuits spread inside and coupled to a onedimensional waveguide in a circuit QED setup. The arrival of a propagating quantum microwave field induces an irreversible change in the population of the internal levels of the absorbers, due to a selective absorption of photon excitations. This design is studied using a formal but simple quantum field theory, which allows us to evaluate the single-photon absorption efficiency for one and many absorber setups. As an example, we consider a particular design that combines a coplanar coaxial waveguide with superconducting phase qubits, a natural but not exclusive playground for experimental implementations. This work and a possible experimental realization may stimulate the possible arrival of "all-optical" quantum information processing with propagating quantum microwaves, where a microwave photodetector could play a key role.

PACS numbers: 42.50.-p, 85.25.Pb, 85.60.Gz
\end{abstract}

Submitted to: Phys. Scr. 


\section{Introduction}

In a recent work [1] we suggested a possible implementation of a photon detector that may also work as a photon counter in the microwave regime. Our proposal builds on previous advances in the field of quantum circuits in two fronts. One is the development of artificial atoms and qubits [2, 3, 4, 5] for quantum computation and quantum information processing, using quantized charge [6, 7, 8, 9], flux [10, 11, 12, 13], or phase [14, 15, 16, 17] degrees of freedom. The other front is the efficient coupling of these elements to microwave guides and cavities conforming the emergent field of circuit quantum electrodynamics (QED) [18, 19, 20]. Without neglecting important advances in intracavity field physics in circuit QED, as we will continue to illustrate below, here we are interested in the physics of propagating quantum microwaves.

As we argued before [1], in order to fully unleash the power of quantum correlations in propagating microwave photonic fields, as may be generated by circuit QED setups, the implementation of efficient photon detectors and counters would be mostly welcomed. The existence of these detectors is implied by almost any sophisticated quantum protocol involving optical photons, be in their coherent interaction with matter [21] or purely all-optical devices [22]. It ranges from the characterization and reconstruction of nonclassical states of propagating light by quantum homodyne tomography [23] to high-fidelity electron-shelving atomic qubit readout [24]. Both examples coming from quantum optics have shown to be influential in the novel field of circuit QED, with the first theoretical [25] and experimental efforts [26] to measure relevant observables of propagating microwaves, and a recent proposal of mesoscopic shelving qubit readout [27]. In spite of these efforts, it will be very hard to overcome the necessity of photon detectors and counters when the emerging field of quantum

microwaves will want to deal with local and remote interqubit/intercavity quantum communication, implementations of quantum cryptography, and other key advanced quantum information protocols [21, 22].

It should be thus no wonder that photon detection and counting become soon a central topic in the field of quantum circuits, where superconducting circuits interact with intracavity and propagating quantum microwaves. So far we have seen the exchange of individual photons between superconducting qubits and quantum resonators [28, 29, 30], the resolution of photon number states in a superconducting circuit [31, the generation of propagating single photons [32], the first theoretical efforts for detecting travelling photons [33, 34], and the nonlinear effects that arise from the presence of a qubit in a resonator [35, 36]. We envision a rich dialogue between intracavity and intercavity physics in the microwave domain, see for example [37, 38, 39], where matter and photonic qubits exchange quantum information in properly activated quantum networks for the sake of quantum information processing.

All efforts towards the implementation of a photodetector for propagating microwaves in circuit QED face a number of challenges, many of which are related to the specific nature of quantum circuits [1]. These are: i) Available cryogenic 

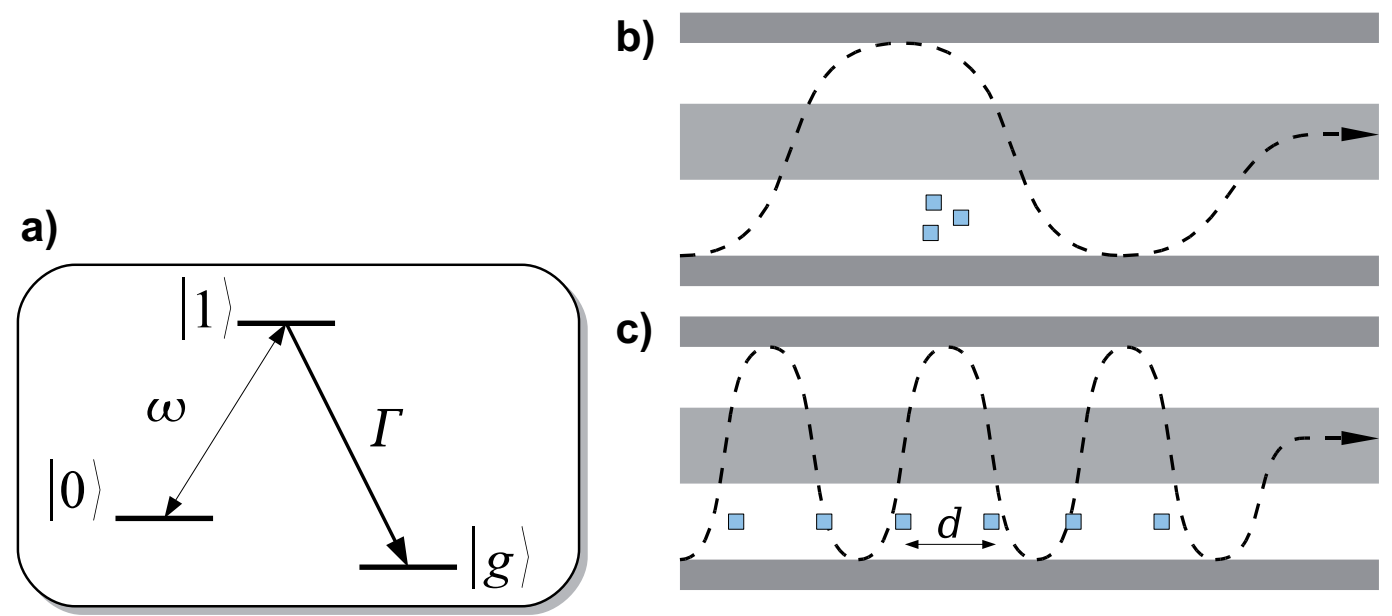

Figure 1. Our photodetector scheme consists of three-level absorbers (a) embedded in a coplanar coaxial microwave guide (long gray stripes). The absorbers (squares) can either be grouped in clusters that are much smaller than a wavelength (b) or regularly spaced (c). As the waveguide provides an effectively one-dimensional setup, the transverse position of the absorbers affects mildly the coupling strength.

linear amplifiers are unable to resolve the few photon regime. ii) Free-space crosssection between microwave fields and matter qubits are known to be small. iii) The use of cavities to enhance the coupling introduces additional problems, such as the frequency mode matching and the compromise between high-Q and high reflectivity. iv) The impossibility of performing continuous measurement without backaction [33], which leads to the problem of synchronizing the detection process with the arrival of the measured field. In a wide sense, the photodetection device has to be passive, being activated irreversibly by the arriving microwave signal. Otherwise, the advanced information that a photon is approaching turns itself into a photodetection device.

Our proposal for a photon detector consists of a very simple setup, a microwave guide plus a number of superconducting circuits that absorb photons [Fig. 1], and is able to circumvent at once the problems describe above. Instead of unitary evolutions, we make use of an irreversible process which maps an excitation of the travelling electromagnetic field (a photon) into an excitation of a localized quantum circuit. By separating this encoding process from the later readout of this information, we avoid the backaction problem coming from continuous measurement. Furthermore, we can compensate different limitations - weak coupling, low efficiency of absorption, photon bandwidth - using no more than a few absorbing elements [Fig. 1 k], where collective effects enhance the detection efficiency.

In this work we develop in great detail the theory underlying our proposal for high-efficiency phodetection [1]. In Sec. 2, we develop an abstract model that consists on a one-dimensional wave guide that transports photons and a number of threelevel quantum systems that may absorb those photons. We will solve analytically the evolution of an incoming wavepacket, studying the time evolution of the full system with 
one or more detecting elements. For a single photon we can compute the absorption efficiency and how it depends on various parameters: detuning of photons from the absorber transition, irreversible decay rate of absorbers, spatial distribution of detectors and temporal profile of photon. In Sec. 3, we go further with this theoretical model by applying it to a particular design where the waveguide is a coplanar coaxial microwave guide, built with superconducting materials, and the absorbing elements are built from superconducting phase qubits. The parameters of the abstract model are matched to the experimental frequencies, impedances and capacitances of the chosen setup, verifying the experimental viability of our model. For completeness, in Sec. 4, we write down the quantum field theory for the microwave guide as it is used and needed in Sec. 2 and Sec. 3. Finally, in Sec. 5, we summarize our results and discuss the experimental challenges and future scopes.

\section{Abstract model and design}

In this section we present in an abstract manner our photodetector design: a onedimensional waveguide transporting photons that can be absorbed by one or more bistable elements that, for the sake of simplicity, will be called indistinctively absorbers or qubits. We will introduce a general mathematical model that uses two fields to describe the quantized waves (photons) and three-level systems for the absorbers. We will study the consequences of this model, including how the joint device may act as a photon detector or photon counting device. This mathematical formalism will serve as a foundation for the developments in Sec. 3, where we will specify the microscopic theory behind the waveguide and the photon absorbers.

\subsection{Master equation}

Following the standard quantum optical master equation formalism, we describe the joint state of the propagating photons and the absorbing elements using a density matrix, $\rho$. This is a Hermitian operator that contains information about the probability distributions of all observables in the system. To the lowest order of approximation the density matrix will evolve according to the master equation

$$
\frac{d}{d t} \rho=-\frac{i}{\hbar}[H, \rho]+\mathcal{L} \rho
$$

Here, the conservative part of the evolution is ruled by the Hamiltonian

$$
H=H_{\text {photon }}+H_{\text {qubit }}+H_{\text {int }},
$$

which contains terms describing the propagation of microwave photons in the guide, the absorbing elements and the interaction between matter (absorbers) and radiation (field), respectively. The first operator contains two propagating fields, $\psi_{r}$ and $\psi_{l}$ moving rightwards and leftwards with group velocity $v$

$$
H_{\text {photon }}=\int\left[\psi_{r}^{\dagger}\left(-i \hbar v \partial_{x}\right) \psi_{r}+\psi_{l}^{\dagger}\left(+i \hbar v \partial_{x}\right) \psi_{l}\right] d x
$$


The second part models one or more discrete quantum elements that we place close to the transmission line. These elements are analogous to the qubits in quantum computing and circuit-QED setups, and will play the role of absorbers, or qubits, enjoying at least three energy levels. The first two, $|0\rangle$ and $|1\rangle$, are metastable and separated by an energy $\hbar \omega$ close to the frequency of the incoming photons

$$
H_{\text {qubit }}=\sum_{i=1}^{N} \hbar \omega|1\rangle_{i}\langle 1| .
$$

Then, there is the interaction between the electromagnetic field and our qubits. We model it with a delta-potential which induces transitions between the qubit states at the same time it steals or deposits photons in the wave guide

$$
H_{\mathrm{int}}=\sum_{i=1}^{N} \int V \delta\left(x-x_{i}\right)\left[\psi_{r}(x)+\psi_{l}(x)\right]|1\rangle_{i}\langle 0| d x+\text { H.c. }
$$

Finally we have included a Liouvillian operator $\mathcal{L}$ which models the decay of the absorbing elements from the metastable state $|1\rangle$ to a third state, $|g\rangle$, and which constitutes the detection process itself. A general second order Markovian model for the decay operator reads

$$
\mathcal{L} \rho=\sum_{i=1}^{N} \frac{\Gamma}{2}\left[2|g\rangle_{i}\langle 1|\rho| 1\rangle_{i}\langle g|-| 1\rangle_{i}\langle 1|\rho-\rho| 1\rangle_{i}\langle 1|\right] .
$$

Note that if we start with a decoupled qubit $(V=0)$ in state $|1\rangle$, the population of this state is depleted at a rate $\Gamma$

$$
\rho(t)=e^{-\Gamma t}|1\rangle\langle 1|+\ldots
$$

\subsection{Non-Hermitian solution}

Let us consider the simple case of one qubit or absorber. The master equation (1) can be written in a more convenient form

$$
\frac{d}{d t} \rho=A \rho+\rho A^{\dagger}+\Gamma|g\rangle\langle 1|\rho| 1\rangle\langle g|
$$

where we have introduced a non-Hermitian operator

$$
A=-\frac{i}{\hbar} H-\frac{\Gamma}{2}|1\rangle\langle 1| \neq A^{\dagger}
$$

The master equation can now be manipulated formally using the "interaction" picture

$$
\rho(t)=e^{A t} \sigma(t) e^{A^{\dagger} t},
$$

with the following equation for $\sigma(t)$,

$$
\frac{d}{d t} \sigma=\Gamma e^{-A t}|g\rangle\left\langle 1\left|e^{A t} \sigma e^{A^{\dagger} t}\right| 1\right\rangle\langle g| e^{-A^{\dagger} t} .
$$

Using the relation $e^{A t}|g\rangle=e^{A^{\dagger} t}|g\rangle=|g\rangle$ we obtain

$$
\frac{d}{d t} \sigma=\Gamma|g\rangle\left\langle 1\left|e^{A t} \sigma e^{A^{\dagger} t}\right| 1\right\rangle\langle g| .
$$


This equation can be solved by splitting into a part that already decayed, $\sigma_{g g} \propto|g\rangle\langle g|$, and all other contributions $\sigma_{\perp}=1-\sigma_{g g}$

$$
\begin{aligned}
\frac{d}{d t} \sigma_{g g} & =\Gamma|g\rangle\langle g| \times \sum_{\alpha, \beta}\left\langle 1\left|e^{A t} \sigma_{\perp} e^{A^{\dagger} t}\right| 1\right\rangle, \\
\frac{d}{d t} \sigma_{\perp} & =0 .
\end{aligned}
$$

Using the initial condition

$$
\sigma_{g g}(0)=0, \sigma_{\perp}(0)=\rho(0)=|\Psi(0)\rangle\langle\Psi(0)|,
$$

we obtain the formal solution

$$
\rho(t)=|\Psi(t)\rangle\langle\Psi(t)|+| g\rangle\langle g| \int_{0}^{t} \Gamma\langle 1 \mid \Psi(\tau)\rangle\langle\Psi(\tau) \mid 1\rangle d \tau,
$$

where we have introduced state $|\Psi(t)\rangle$, evolving from initial state $|\Psi(0)\rangle$ according to the lossy Schrödinger equation

$$
\frac{d}{d t}|\Psi(t)\rangle=A|\Psi(t)\rangle \equiv-\frac{i}{\hbar} \bar{H}|\Psi(t)\rangle
$$

Note that the probability of the qubit irreversibly capturing a photon is given by

$$
\begin{aligned}
P(0 \rightarrow 1 \rightarrow g) & =\operatorname{Tr}(\rho|g\rangle\langle g|) \\
& =1-\operatorname{Tr}[|\Psi(t)\rangle\langle\Psi(t)|] \\
& =1-\|\Psi\|^{2} .
\end{aligned}
$$

\subsection{Model equations for a single absorber}

Following the previous reasoning, our goal is to solve the lossy Schrödinger equation

$$
i \frac{d}{d t}|\Psi\rangle=\bar{H}|\Psi\rangle
$$

ruled by the non-Hermitian operator

$$
\bar{H}=H_{\text {photon }}+H_{\text {qubit }}+H_{\text {int }}-\sum_{i} \frac{\hbar \Gamma}{2}|1\rangle_{i}\langle 1| \text {. }
$$

In order to describe the detection process, our initial condition $|\Psi(0)\rangle$ will contain the incoming photons plus all absorbers in the ground state $|0\rangle$. The probability that an absorber captures a photon and changes from state $|0\rangle$ to $|g\rangle$ is given by the loss of norm shown in Eq. (19).

Let us consider an incident photon coming from the left with energy $E=\hbar|k| v$. The state of the system will be given by [40]

$$
\begin{aligned}
|\Psi\rangle & =\int\left[\xi_{r}(x, t) \psi_{r}^{\dagger}(x)+\xi_{l}(x, t) \psi_{l}^{\dagger}(x)\right]|v a c, 0\rangle+ \\
& +e_{1}|v a c, 1\rangle .
\end{aligned}
$$

Here $\psi_{r, l}^{\dagger}(x)|v a c, 0\rangle$ is the state of a photon created at position $x$ and moving either to the right or to the left, while the absorber is in metastable state $|0\rangle$. Also, $\xi_{r}(x, t)$ and $\xi_{l}(x, t)$ represent the wavefunction of a single photon moving to the right and to the 


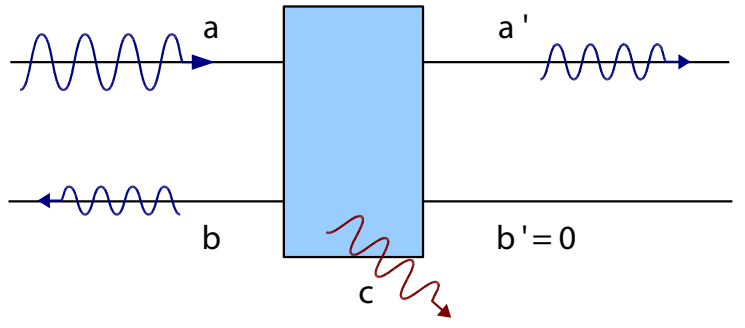

Figure 2. An incident photon, moving rightwards, interacts with an absorbing element. Out of the original amplitude of the field, $a$, a component is transmitted, $a^{\prime}$, another component is reflected, $b$, and finally with some probability, $|c|^{2}$, the system absorbs a photon and changes state.

left, respectively, while $|v a c, 1\rangle$ is a state with no photons and the absorber excited to unstable level $|1\rangle$. Note that thanks to the relation (16) we do not need to explicitely include the population of state $|g\rangle$. We only have to solve the Schrödinger equation (20) using a boundary condition that represents a photon coming from the left and an inactive absorber

$$
\xi_{r}\left(x>0, t_{0}\right)=\xi_{l}\left(x, t_{0}\right)=0, e_{1}\left(t_{0}\right)=0,
$$

and compute the evolution of the photon amplitude, $\xi_{r, l}(x, t)$, the excited state population $e_{1}(t)$ and the resulting photon absorption probability (19).

After decomposing the wave equation into the left $(x<0)$ and right $(x>0)$ halves of space, and replacing the potential $\delta\left(x-x_{1}\right)$ with an appropriate boundary condition at $x_{1}=0$, one obtains the Schrödinger equation for the absorber

$$
\begin{aligned}
i \partial_{t} e_{1} & =\frac{V}{2 \hbar}\left[\xi_{r}\left(0^{+}\right)+\xi_{r}\left(0^{-}\right)+\xi_{l}\left(0^{+}\right)+\xi_{l}\left(0^{-}\right)\right], \\
& +(\omega-i \Gamma) e_{1}
\end{aligned}
$$

and four equations for the photon,

$$
\begin{array}{ll}
i \partial_{t} \xi_{r}(x, t) & =-i v \partial_{x} \xi_{r}(x, t), \quad x \neq 0, \\
i \partial_{t} \xi_{l}(x, t) & =+i v \partial_{x} \xi_{l}(x, t), \quad x \neq 0, \\
0 & =-i \hbar v\left[\xi_{r}\left(0^{+}, t\right)-\xi_{r}\left(0^{-}, t\right)\right]+V e_{1}(t), \\
0 & =+i \hbar v\left[\xi_{l}\left(0^{+}, t\right)-\xi_{l}\left(0^{-}, t\right)\right]+V e_{1}(t) .
\end{array}
$$

We introduce new variables $a, b, a^{\prime}$ and $b^{\prime}$ describing the amplitude of the fields on both sides of an absorber [Fig. 2],

$$
\begin{array}{ll}
a(t)=\xi_{r}\left(0^{-}, t\right), & a^{\prime}(t)=\xi_{r}\left(0^{+}, t\right), \\
b(t)=\xi_{l}\left(0^{-}, t\right), & b^{\prime}(t)=\xi_{l}\left(0^{+}, t\right) .
\end{array}
$$

Two of these variables can be solved from the initial conditions

$$
\begin{aligned}
& a(t)=\xi_{r}\left(0^{+}, t\right)=\xi_{r}\left(-v\left(t-t_{0}\right), t_{0}\right) \\
& b^{\prime}(t)=0,
\end{aligned}
$$


while the rest are extracted from the boundary conditions

$$
a^{\prime}(t)=a(t)+\frac{V}{i \hbar v} e_{1}(t), b(t)=\frac{V}{i \hbar v} e_{1}(t) .
$$

We have expressed all unknowns in terms of $e_{1}(t)$. The population of the excited state now satisfies

$$
i \partial_{t} e_{1}=\left(\omega-i \Gamma-i \frac{V^{2}}{\hbar^{2} v}\right) e_{1}+\frac{V}{\hbar} a(t),
$$

which contains the original frequency, $\omega$, and an imaginary component for the decay of the three-level system, $\Gamma$, enhanced by the interaction with the transmission line $V^{2} / \hbar^{2} v$. Solving for $e_{1}$, we find

$$
\begin{aligned}
e_{1}(t) & =-i \frac{V}{\hbar} \int_{0}^{t} e^{-\tilde{\Gamma}(t-\tau)} a(\tau) d \tau, \\
\tilde{\Gamma} & =\Gamma+\frac{V^{2}}{\hbar^{2} v}+i \omega,
\end{aligned}
$$

in terms of the incoming beam (27).

Using the expression of $e_{1}(t)$ one may compute the reflected and transmitted components of a microwave beam, to find that the total intensity is not conserved. Rather, we have an absorbed component that is "stolen" by the three-level system to perform a transition from the $|0\rangle$ to $|g\rangle$ states via $|1\rangle$. The balance equations are thus [Fig. 2]

$$
|a|^{2}+\left|b^{\prime}\right|^{2}=\left|a^{\prime}\right|^{2}+|b|^{2}+|c|^{2} .
$$

If we consider a photon with a finite duration, $T$, the detection efficiency will be defined as the fraction of the wavepacket that was absorbed

$$
\alpha=\frac{\int_{0}^{T}|c(t)|^{2} d t}{\int_{0}^{T}\left[|a(t)|^{2}+\left|b^{\prime}(t)\right|^{2}\right] d t} .
$$

For the purposes of photodetection and photon counting we would like this value to reach the maximum $\alpha=1$.

\subsection{Stationary solutions for one qubit}

To understand the photon absorption process we will study a continuous monochromatic beam which is slowly switched on,

$$
a(t)=\exp \left[\left(-i \omega_{0}+\sigma\right) t\right] \quad t \leq 0, \sigma>0 .
$$

Taking the limits $t_{0} \rightarrow-\infty$ and $\sigma \rightarrow 0^{+}$in this precise order, we obtain

$$
\begin{aligned}
e_{1} & =-i \frac{V}{\hbar} \int_{-\infty}^{t} \exp \left[-\tilde{\Gamma}(t-\tau)+\left(-i \omega_{0}+\sigma\right) \tau\right] \\
& =-i \frac{V / \hbar}{\Gamma+\frac{V^{2}}{\hbar^{2} v}+i\left(\omega-\omega_{0}\right)} e^{-i \omega_{0} t} .
\end{aligned}
$$

We will introduce a unique parameter to describe the photodetection process,

$$
\gamma=\frac{\hbar v}{V^{2}} \hbar\left[\Gamma+i\left(\omega-\omega_{0}\right)\right],
$$


which includes both a renormalization of the decay rate and a small imaginary component associated to the detuning. With this parameter the solution reads

$$
\begin{aligned}
& e_{1}(t)=-i \frac{\hbar v}{V} \frac{1}{1+\gamma} e^{-i \omega_{0} t} \\
& a^{\prime}(t)=\left[1-\frac{1}{1+\gamma}\right] e^{-i \omega_{0} t}, \\
& b(t)=-\frac{1}{1+\gamma} e^{-i \omega_{0} t} .
\end{aligned}
$$

If we work in the perfectly tuned regime, $\omega=\omega_{0}$, the decay rate $\gamma$ becomes real and the absorption rate is

$$
\begin{aligned}
\alpha & =-2 \operatorname{Re}\left(a^{*} b\right)-2|b|^{2} \\
& =\frac{2}{1+\gamma}-\frac{2}{(1+\gamma)^{2}} \\
& =\frac{2 \gamma}{(1+\gamma)^{2}}=2 b(1-b) .
\end{aligned}
$$

This value achieves a maximum of $50 \%$ efficiency or $\alpha=1 / 2$ at the values $b=1 / 2$, $\gamma=1$. We think that the limit of $50 \%$ in the photodetection efficiency is fundamental and related to the Zeno effect, expressing the balance of quantum information between, see Fig. 1a, the reversible absorption of the photon in the first (left) transition channel and the irreversible absorption in the second (rigth) one.

\subsection{Transfer matrix}

We can derive the long wavepacket or quasi-stationary solution in a slightly different manner. Note that for infinitely long wavepackets the population of the excited state is determined by the fields on both sides

$$
\begin{aligned}
e_{1} & =\frac{1}{\left(\omega_{0}-\omega\right)+i \Gamma} \frac{V}{2 \hbar}\left[a+a^{\prime}+b+b^{\prime}\right] \\
& =\frac{\hbar v}{i V \gamma}\left[a+a^{\prime}+b+b^{\prime}\right] .
\end{aligned}
$$

With this the boundary conditions in Eq. (28) transform into a set of equations that only involves the incoming and outgoing fields,

$$
\begin{aligned}
& 0=a^{\prime}-a+\frac{1}{2 \gamma}\left(a+a^{\prime}+b+b^{\prime}\right), \\
& 0=b^{\prime}-b-\frac{1}{2 \gamma}\left(a+a^{\prime}+b+b^{\prime}\right) .
\end{aligned}
$$

In terms of the matrix and vectors

$$
A=\left(\begin{array}{cc}
1 & 1 \\
-1 & -1
\end{array}\right), x=\left(\begin{array}{l}
a \\
b
\end{array}\right), x^{\prime}=\left(\begin{array}{l}
a^{\prime} \\
b^{\prime}
\end{array}\right),
$$

we can write

$$
\left(1+\frac{1}{2 \gamma} A\right) x^{\prime}=\left(1-\frac{1}{2 \gamma} A\right) x .
$$


Multiplying everything by $(1-A / 2 \gamma)$ and using $A^{2}=0$ gives $x^{\prime}=(1-A / \gamma) x$. This amounts to a relation between the fields to the right and to the left of the absorber

$$
\left(\begin{array}{l}
a^{\prime} \\
b^{\prime}
\end{array}\right)=T\left(\begin{array}{l}
a \\
b
\end{array}\right)
$$

given by the scattering matrix

$$
T=\left(\begin{array}{cc}
1-\frac{1}{\gamma} & -\frac{1}{\gamma} \\
\frac{1}{\gamma} & 1+\frac{1}{\gamma}
\end{array}\right)=1-\frac{1}{\gamma} A .
$$

Note that the value $\gamma$ introduced before (35) only depends on the properties of the qubit, the interaction between the qubit and the line and the group velocity. We have thus

elaborated a rather compact and easily generalizable scheme for studying the scattering and absorption of photons by the individual absorbers.

\subsection{General absorption formula}

Using the transfer matrix formalism we can compute the fraction of absorbed photons. The only requirement to obtain a general formula is that the scattering matrix remains the same under inversion

$$
\left(\begin{array}{l}
b \\
a
\end{array}\right)=T\left(\begin{array}{l}
b^{\prime} \\
a^{\prime}
\end{array}\right)
$$

This is reasonable: from Fig. 2 we can conclude that $b$ and $a^{\prime}$ have the same role, just like $b^{\prime}$ and $a$. Using the relations $b=T_{01} a^{\prime}$ and $a=T_{11} a^{\prime}$, the absorbed fraction becomes

$$
\alpha=1-\frac{\left|a^{\prime}\right|^{2}+|b|^{2}}{|a|^{2}}=1-\frac{1}{\left|T_{11}\right|^{2}}\left(1+\left|T_{01}\right|^{2}\right) .
$$

This is consistent with the single absorber case

$$
T_{11}=\frac{1+\gamma}{\gamma}, T_{01}=-\frac{1}{\gamma}
$$

in which we recover

$$
\alpha=1-\frac{\gamma^{2}}{(1+\gamma)^{2}}\left(1+\frac{1}{\gamma^{2}}\right)=\frac{2 \gamma}{(1+\gamma)^{2}},
$$

as expected.

\subsection{Multiple absorbers}

If we have more than one absorbing element at positions $x_{1}, x_{2} \ldots$, we will use the same formula for the absorption efficiency (150), but with transmission matrix

$$
T_{\text {total }}=T_{1} \Phi\left(x_{2}-x_{1}\right) T_{2} \Phi\left(x_{3}-x_{2}\right) \cdots T_{N}
$$

Here, $T_{i}$ describes the absorption properties of a given absorber and thus depend on the value of $\gamma_{i}$, while the phase matrix $\Phi(x)$ is given by

$$
\Phi(x)=\left(\begin{array}{cc}
e^{i \theta(x)} & 0 \\
0 & e^{-i \theta(x)}
\end{array}\right)
$$



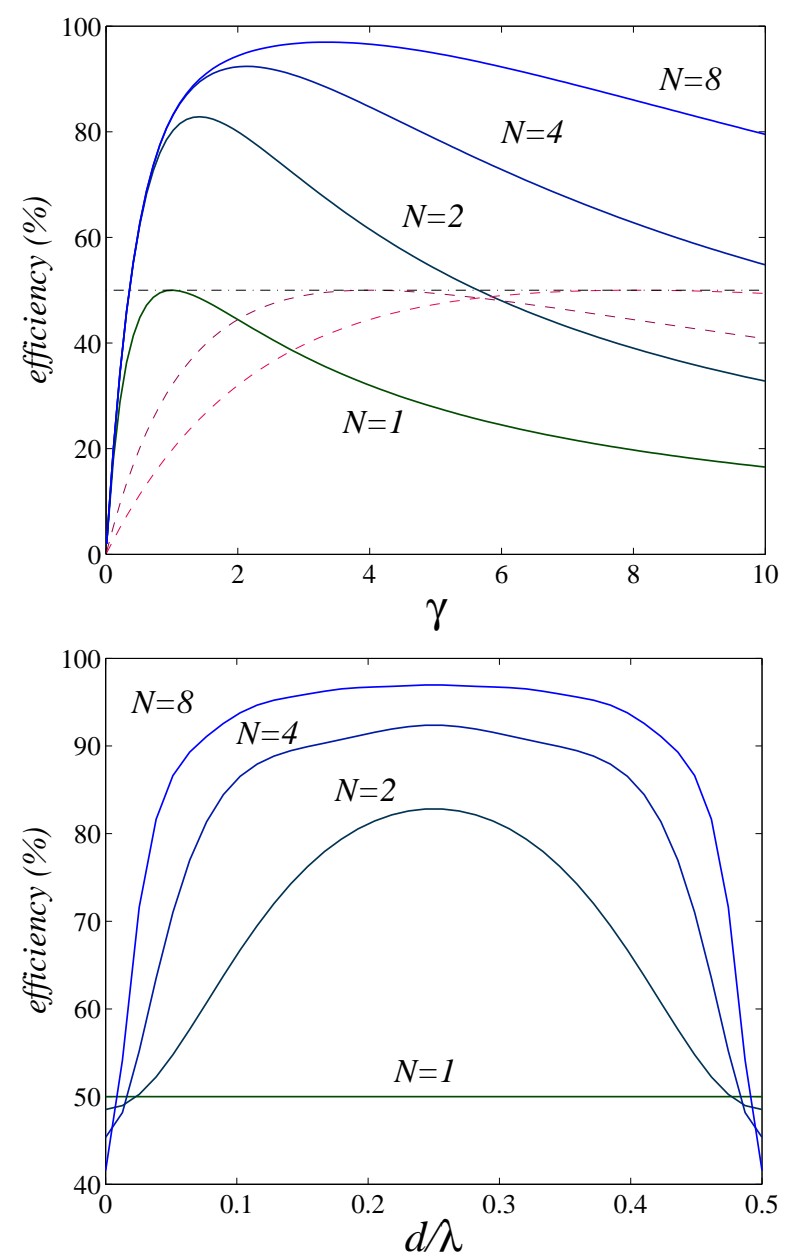

Figure 3. Detection efficiency. (Top) Absorption probability vs. effective decay rate for a setup with $N=1,2,4$ and 8 qubits (black, green, blue, red) either in cluster (dashed) or array (solid) regime. In the first case, where qubits are close together, the efficiency is fundamentally limited to $50 \%$, while in the other case there is no upper limit. In both cases, having more qubits allow us to use weaker couplings or stronger decay rates. (Bottom) Detection efficiency versus the separation $d$ of the absorbing elements for an array of periodically distributed Josephson junctions. All curves have been produced for resonant qubits.

and contains the accumulated phase of the electromagnetic wave when travelling between consecutive absorbers

$$
\theta(x)=\frac{2 \pi}{\lambda} x=\frac{\omega_{0}}{v} x .
$$

\subsection{Analysis of the efficiency}

The relation (47) is valid for one, two and more absorbing elements, placed at different positions. If we have a single element, we have already found the absorbed fraction (52). As shown in Fig. 3, this quantity is upper bounded by $50 \%$ which is reached for resonant qubits with $\gamma=1$. This means that a single absorbing element, without knowing the 

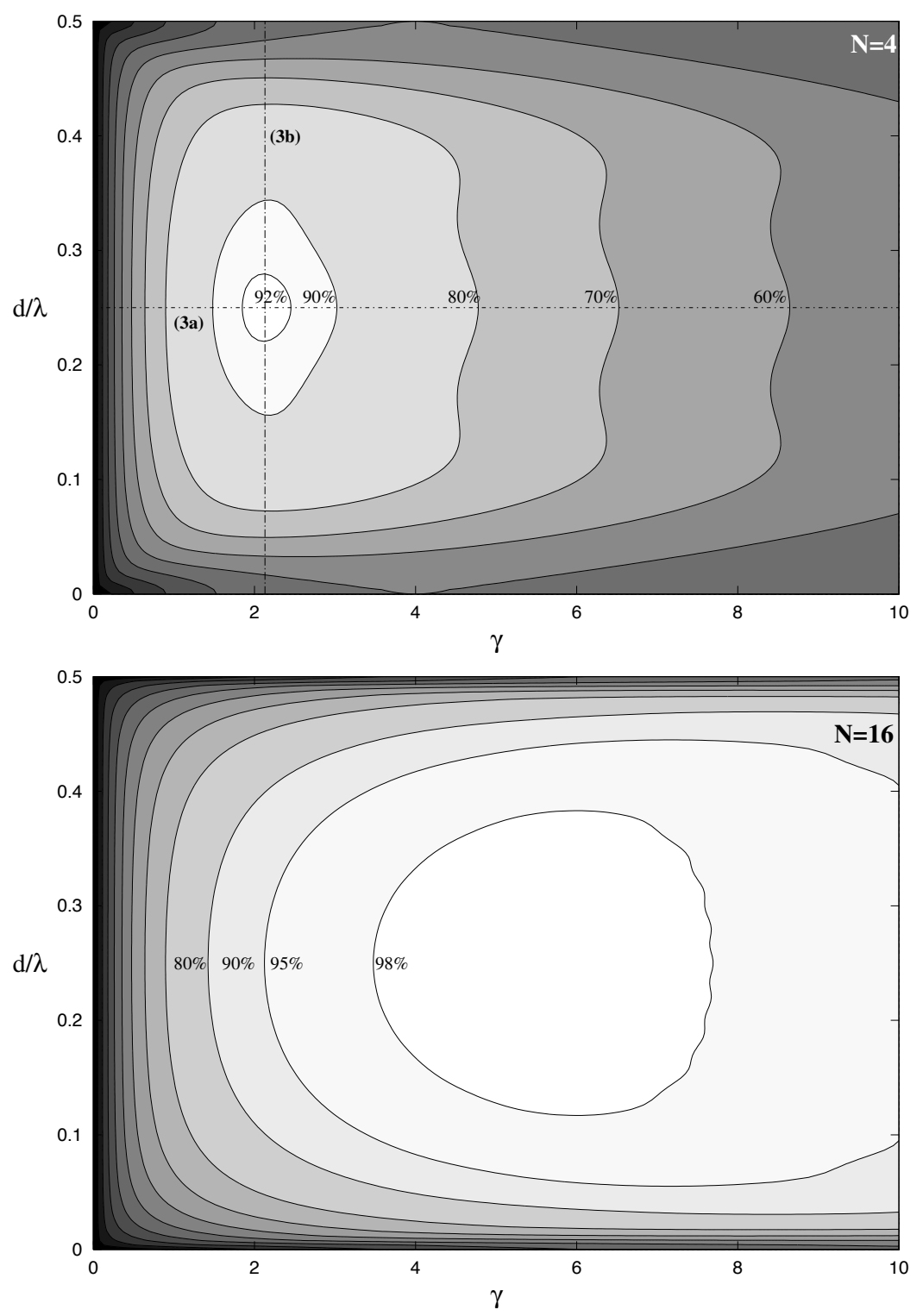

Figure 4. Dependency of the detector efficiency on the parameter $\gamma$ and on the separation between absorbers, in units of the microwave wavelength, $d / \lambda$. We have chosen $N=4$ (above) and $N=16$ (below). Note that the maximum gets broader with $N$, but it is always achieved at $d=\lambda / 4$. The solid lines denote the cuts corresponding to the plots in Fig. 3

arrival time and no particular external modulation, can only detect in average half of the incoming photons.

The situation does not improve if we place $N$ absorbing elements close together [Fig. 1b]. When the wavelength of the microwave field is large compared to the size of the absorber cluster, the total transfer matrix is given by

$$
T=T_{N}^{N}=\left(1+\frac{1}{\gamma} A\right)^{N}=1+\frac{N}{\gamma} A .
$$

This is just the same matrix with a smaller effective decay rate $\gamma / N$, and the total 
efficiency remains limited to $50 \%$,

$$
\alpha_{N}=\frac{2(\gamma / N)}{[1+\gamma / N]^{2}} \leq \frac{1}{2} .
$$

We can do much better, though, if we place a few absorbing elements separated by some distance $d$ [Fig. 1 $\mathrm{k}]$. In this case the total transfer matrix is given by

$$
T_{N}(d)=\left[T_{1}\left(\begin{array}{cc}
e^{i \omega_{0} d / v} & 0 \\
0 & e^{-i \omega_{0} d / v}
\end{array}\right)\right]^{N} .
$$

The total efficiency now depends on two variables, $\gamma$ and the phase $\theta=\omega_{0} d / v$ or the separation between absorbers, $d$. As Fig. 4 shows, the optimal value of the phase is $\theta=\pi / 2$, corresponding to $\lambda / 4$ separation and, in this case, the total efficiency is no longer limited. For instance, as shown in Fig. 3 for two and three qubits on-resonance the efficiency can reach $80 \%$ and $90 \%$, respectively. Not only it grows, but it does so pretty fast.

An interesting feature is that, as we increase the number of qubits, the absorbed fraction becomes less sensitive to the qubit separation, which allows for more compact setups than one would otherwise expect. For instance, in Fig. 5 we show the total size of a setup computed for a fixed detection efficiency and a given number of qubits. Since the optimal separation behaves as $d \sim 1 / N$, the total system size for a fixed detector efficiency, $78 \%, 80 \%$ and so on, remains bounded, even for large number of qubits. Moreover, as one increases the number of qubits, the efficiency grows rapidly. We can define the value

$$
\alpha_{\infty}(\mathcal{L})=\lim _{N \rightarrow \infty} \max _{\gamma} \alpha(\mathcal{L}, N, \gamma, d=\mathcal{L} / N),
$$

which gives an idea of what is the maximum detection efficiency for a given circuit size. The value shown in Fig. 5b approaches the limit of $100 \%$ quite fast and gives us an idea of the minimal size of a detector which is needed to obtain a given efficiency.

\subsection{Robustness against imperfections}

There are many factors that will condition the actual efficiency of a photodetector. Some of them will have to be discussed later on in the context of the proposed implementation, but others can be analyzed already with the present theory.

The first source of errors that one may consider are systematic differences in the fabrication and tuning of the absorbing elements. These fluctuations are currently unavoidable, and may even evolve through the lifetime of a setup, due to changes in the temperature, fluctuations of impurities, among others. These systematic errors could be modeled by random perturbations in the parameters of the three-level systems, either due to inhomogeneous broadening (different frequencies $\omega_{i}$ ), inhomogeneous decay rates (different $\Gamma_{i}$ ) or changes in the coupling strengths $\left(V_{i}\right)$. However, since the scattering of photons is described by a single parameter $\gamma_{i}$ per qubit, it is more convenient to model the errors as random changes in these values. 


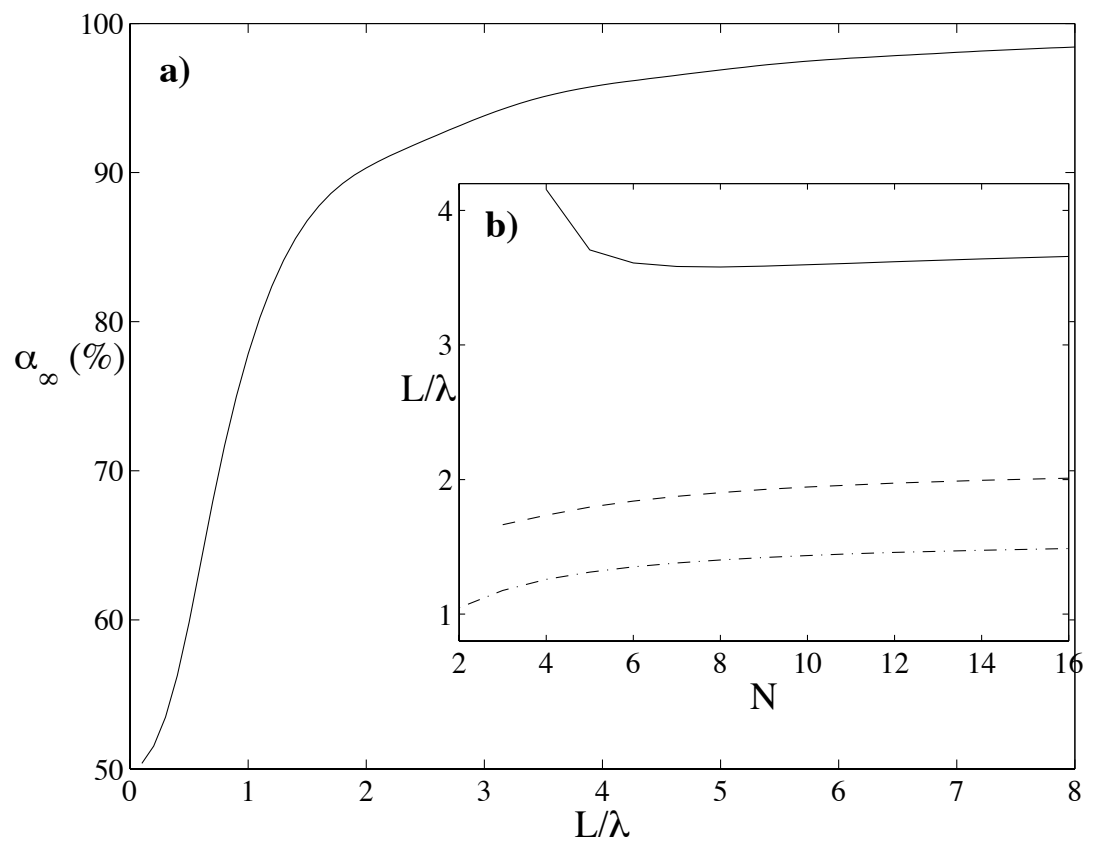

Figure 5. Optimal separation of detectors. (a) Asymptotic detector efficiency $\alpha_{\infty}(L)$ as a function of the setup size $L$, and computed as in Eq. (59). (b) Minimal size $L$ of a setup with $N$ qubits required to attain a detection efficiency $\alpha=78 \%$ (dash-dot), $85 \%$ (dashed) or $\alpha=92 \%$ (solid).

We have performed a numerical study using a constant probability distribution, $P(\gamma)=1 / 2 \sigma_{\gamma} \bar{\gamma}$ for $|\gamma-\bar{\gamma}| \leq \sigma_{\gamma} \bar{\gamma}$, with a maximum relative error $\sigma_{\gamma}$ that ranges from 0 up to $20 \%$. Using 10000 random realizations with different parameters $\left\{\gamma_{i}\right\}_{i=1}^{N}$, we made statistics of the detector efficiency, $\alpha$. The results are shown in Fig. 6, where we plot the standard deviation of the maximum detection efficiency, $\sigma_{\alpha}$, given by

$$
\sigma_{\alpha}=\sqrt{\left\langle\left\langle\alpha^{2}\right\rangle\right\rangle-\langle\langle\alpha\rangle\rangle^{2}}
$$

where the double bracket $\langle\langle\cdots\rangle\rangle$ denotes average over the random instances. According to our results, the maximum efficiencies for $N=4,8,16$, and 32 are $\alpha=$ $92.4 \%, 97 \%, 98.9 \%$ and $99 \%$, and in the worst case of $20 \%$ maximum systematic errors, where one standard deviation implies an efficiency decrease of $0.7 \%$ for 4 absorbers and $0.1 \%$ for 32 . In other words, we find up to two orders of magnitude smaller errors in the detector than in the fabrication.

\subsection{Detector bandwidth and dephasing}

There are two other sources of error which are intimately connected. One is the bandwidth of the incoming photons, $\Delta \omega$, which can be related to the length of the photon wavepacket, $\tau \sim 1 / \Delta \omega$. Since the detector efficiency depends on $\gamma \propto \Gamma+i\left(\omega-\omega_{0}\right)$, which contains the detuning between the photon and qubit frequencies, some spectral components may only be weakly detected. To solve this problem we need the detector 


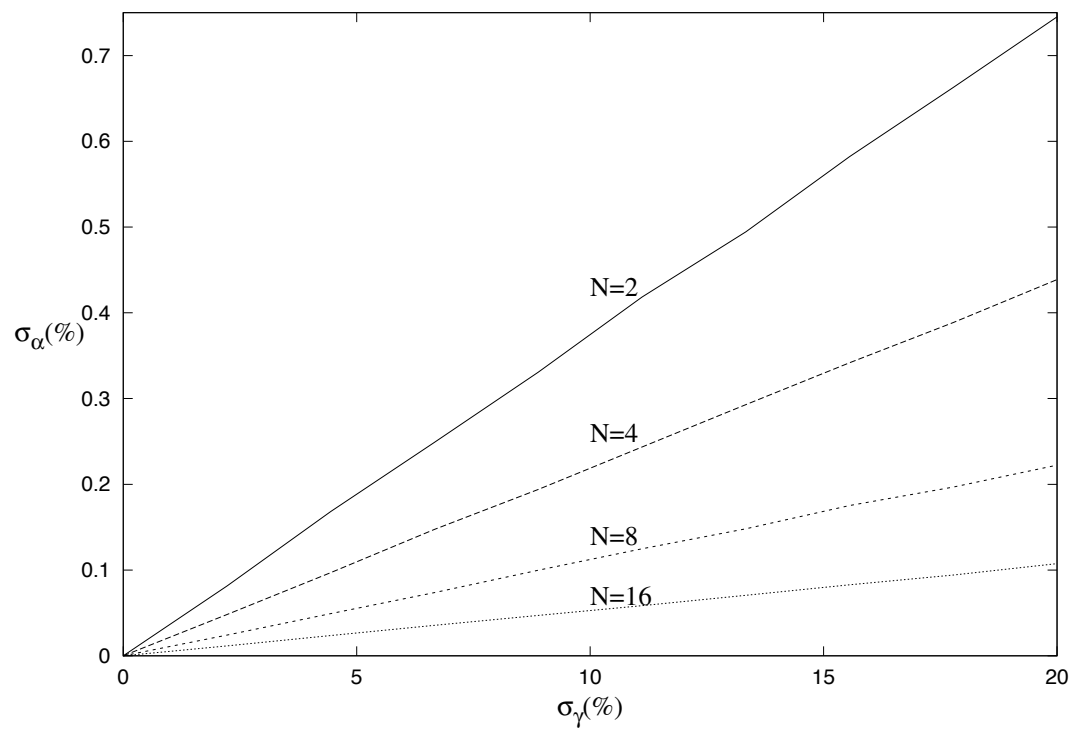

Figure 6. Sensitivity of the detector to systematic errors in the absorbers. We plot the standard deviation of the absorption efficiency, $\sigma_{\alpha}$, in percentiles, vs. the maximum systematic random error, $\sigma_{\gamma}$, averaged over 10000 random samples with $N=4,8,16$ and 32 qubits (top to bottom).

efficiency to be weakly sensitive to variations in the detuning of the photon, $\delta=\omega-\omega_{0}$, that is we need a broadband detector.

To estimate the bandwidth of the photodetector we have applied the following criterion. We compute the optimal value of $\gamma_{o p t}$ for which the detection happens at maximum efficiency with zero detuning, $\delta=\omega-\omega_{0}=0$. We then look for the two values of the detuning at which $\gamma=\gamma_{o p t}(1+1 i \delta / \Gamma)$ causes a reduction of the efficiency of a given percentage. As Fig. 7 $\mathrm{b}$ shows, the bandwidth grows with a power law $N^{\nu}$, with an exponent which is unfortunately not too large. However, Fig. 7a shows that the detuning may actually increase the efficiency, probably indicating that the previous analysis is too limited and that the detector design may involve optimizing both $\Gamma$ and $\delta$ not only for achieving a certain efficiency, but also to increase the efficiency.

Another related problem is dephasing. As we will discuss later on, quantum circuits are affected by $1 / \mathrm{f}$ noise. Part of this noise can be understood as oscillating impurities that change the electromagnetic environment of the absorbers, and thus the relative energies of the 0 and 1 states. This error source is modeled with a term $\epsilon \sigma^{z}=\epsilon(|1\rangle\langle 1|-| 0\rangle\langle 0|)$, where $\epsilon$ is a random variable - either classical, or quantum, from a coupling with the environment-. When one averages over the different noise realizations, the result is decoherence.

We can get rid of the noise for each realization using a unitary operator $U(t) \sim$ $\exp \left(-i \int_{0}^{t} \epsilon \sigma^{z} d \tau / \hbar\right)$. When we use this operator to simplify the Schrödinger equation, creating the equivalent of an interaction picture, the result is that we can translate the 

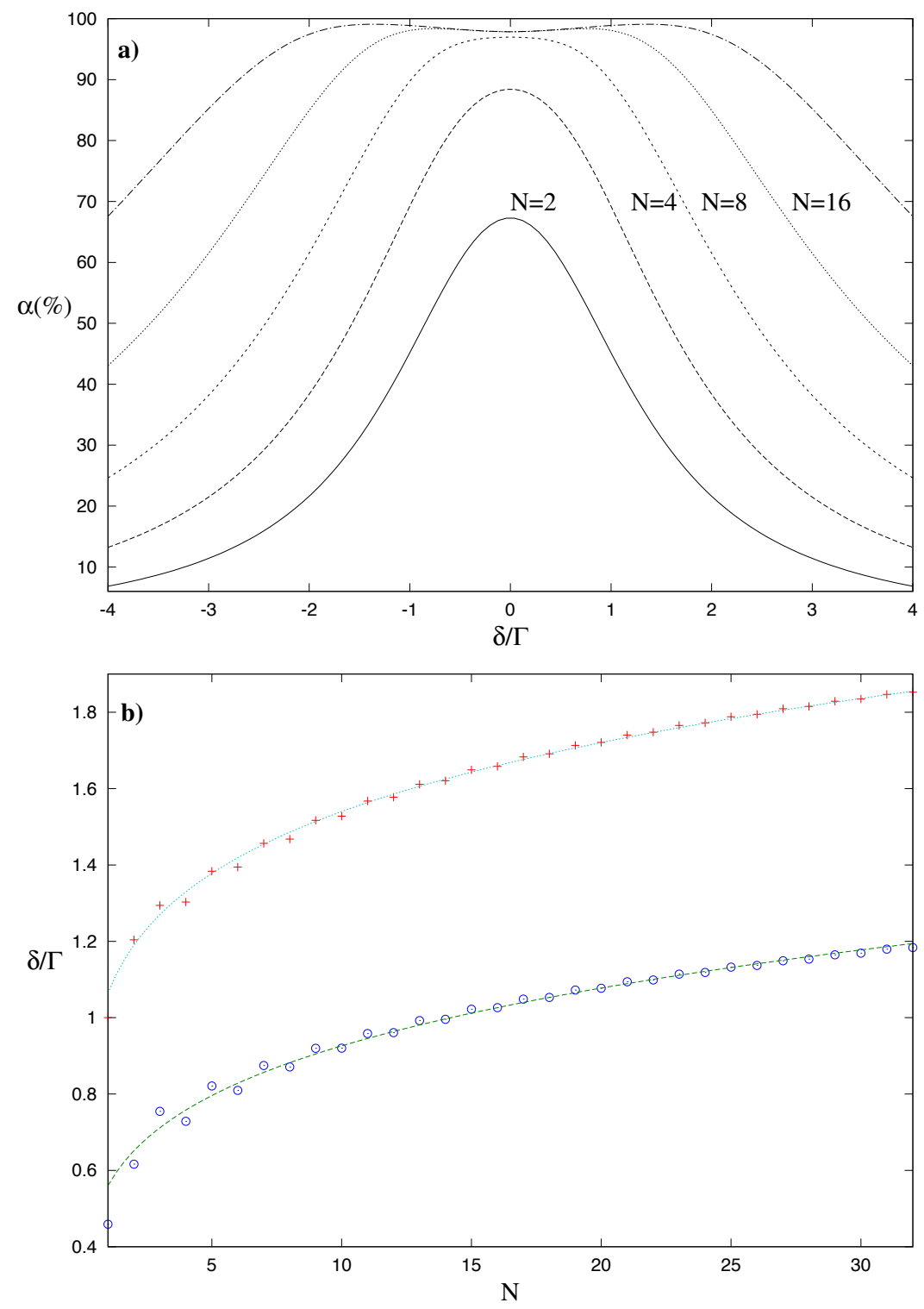

Figure 7. (a) Change of the detector efficiency as we introduce a detuning between the photon and absorber frequencies. (b) Bandwidth of the photo detector vs. the number of absorbers. Given the optimal working point $\gamma_{o p t}$ which maximizes the detection efficiency at zero detuning, $\delta=\omega-\omega_{0}$, we look for the value of the detuning such that the efficiency at $\gamma=\gamma_{o p t}(1+1 i \delta)$ is reduced by $5 \%$ (circles) or $20 \%$ (crosses). The solid lines are fits to $N^{0.16}$ and $N^{0.2}$.

accumulated random phase,

$$
\phi(t, \epsilon)=\int_{0}^{t} \epsilon d \tau / \hbar,
$$

into the coupling

$$
H_{\text {int }} \sim V \int \delta(x)\left[e^{i \phi_{\epsilon}} \psi_{r}+e^{i \phi_{\epsilon}} \psi_{l}\right]|1\rangle\langle 0|+\text { H.c. }
$$

In other words, from the point of view of the absorber it is as if the incoming photon was 
affected by a random, but slowly changing phase $\dagger$ that may either shift the frequency of the photons or broaden their spectral distribution. In either case, if the photodetector has a large enough bandwidth we may expect just a minor change in the detector efficiency.

\section{Model implementation: microwave guide with phase qubits}

In this section we detail a possible implementation of our scheme which is based on elementary circuits found in today's experiments with superconducting qubits. We will show how our previous theory relates to the mesoscopic physics of these circuits and compute expressions for the relevants parameters, $v, V, \gamma, \ldots$ in terms of the properties of these circuits.

For the waveguide we will consider a coaxial planar microwave guide such as the ones employed to manipulate and couple different qubits [19, 41] and described in detail in Sec. 4. Note, however, that unlike in Ref. [19 our waveguide will not be cut at the borders and it will not form a resonating cavity. For the bistable elements we will consider a superconducting qubit, the so called "phase qubit" or "current-biased Josephson junction" (CBJJ), which has a set of metastable levels that, by absorption and emission of photons, may decay to a different, macroscopically detectable current state.

\subsection{Current-biased Josephson junction}

As mentioned before, our detection element will be a CBJJ. The model for this circuit is shown in Fig. 8, there is a Josephson junction shunted by an current source which can be modeled by a very big impedance. The bias current $I$ causes a tilting of the energy potential in the junction, creating metastable regions with a finite number of energy levels, that tunnel quantum-mechanically outside the barrier.

The quantization of this circuit renders a simple Hamiltonian [42]

$$
H=\frac{1}{2 C_{J}} Q^{2}+U(\phi)
$$

expressed in terms of the charge in the junction and the flux $\phi$, at a node of the circuit [Fig. 8]. The Hamiltonian contains the usual capacitive energy, expressed in terms of the large capacitance of the junction, $C_{J}$, and a potential energy due to the inductive elements. Modeling the current source as a large inductor with a total flux that supplies a constant current, $\tilde{\Phi} / L_{J}=I$, we obtain a highly anharmonic potential

$$
U(\phi)=-I_{0} \varphi_{0} \cos \left(\phi / \varphi_{0}\right)-I \phi \text {. }
$$

Note that even though the actual flux quantum is $\phi_{0}=h / 2 e$, in order to avoid $2 \pi$ factors everywhere it is convenient to work with $\varphi_{0}=\phi_{0} / 2 \pi$.

The quantization of this model corresponds to imposing the usual commutation relations between the canonically conjugate variables, the flux $\phi$ and the charge $Q$, that

$\ddagger$ Remember that the noise source is $1 / \mathrm{f}$ and dominated by low frequencies 

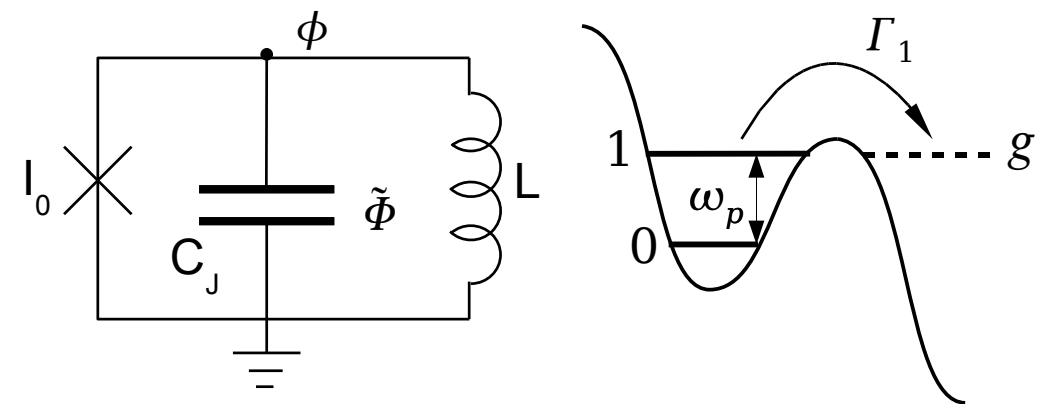

Figure 8. (left) Scheme for a current-biased Josephson junction. (right) Energy levels. There are $N_{s}$ metastable energy levels with anharmonic frequencies.

is $[\phi, Q]=i \hbar$. Given the relevance of the anharmonic terms in Eq. (64) it soon becomes evident the convenience of working in the number-phase representation $\phi=\varphi_{0} \times \theta$, and $Q=2 e \times N$ with operators that satisfy $[\theta, N]=i$. In these variables the Hamiltonian becomes

$$
H=E_{C} N^{2}-I_{0} \varphi_{0}\left[\cos (\theta)+\frac{I}{I_{0}} \theta\right],
$$

with the junction charging energy

$$
E_{C}=\frac{(2 e)^{2}}{2 C_{J}}=\frac{\hbar^{2}}{2 \varphi_{0}^{2} C_{J}} .
$$

\subsection{Harmonic approximation}

When the bias current $I$ is very close to the critical current $I_{0}$, we have the situation in Fig. 8, in which the junction develops a metastable, local minimum of the potential at $\theta$ close to $\pi / 2$. It is then customary to approximate the potential by a cubic polynomial and describe the dynamics semiclassically, with a coherent component that describes the short-time oscillations around the local minimum and a decay rate to the continuum of charge states which are outside this unstable minimum.

The semiclassical limit is characterized by just two numbers, the plasma frequency of the phase oscillations around the minimum

$$
\omega_{p}=\sqrt{\frac{I_{0}}{4 \varphi_{0} C_{J}}}\left[1-\left(\frac{I}{I_{0}}\right)^{2}\right]^{1 / 4}
$$

and the barrier height

$$
\Delta U=\frac{2 \sqrt{2}}{3} \sqrt{1-\frac{I}{I_{0}}},
$$

that prevents tunnneling outside this minimum. Using semiclassical methods it is possible to estimate the number $\sim N_{s}=\Delta U / \hbar \omega_{p}$ of metastable states in this local minimum, and approximate their energy levels,

$$
E_{n} / \hbar=n \omega_{p}+\omega_{n}^{a n h}-i \Gamma_{n},
$$


including an anharmonic component, $\omega_{n}^{a n h}$, and an imaginary part which is the rate at which the state decays into the continuum

$$
\Gamma_{n}=\omega_{p} \frac{\left(432 N_{s}\right)^{n+1 / 2}}{\sqrt{2 \pi} n !} e^{-36 N_{s} / 5} .
$$

In this work, we use the fact that for two consecutive levels these rates can be very different, $\Gamma_{n+1} / \Gamma_{n} \sim 1000$. If the decay rate of the first level $\Gamma_{1}$ is large enough while still keeping $\Gamma_{0}$ small, we can treat the levels $|0\rangle$ and $|1\rangle$ as the two levels of our absorbing element, the continuum playing the role of the detectable states $|g\rangle$. With this we have now the parameters $\omega=\omega_{p}$ and $\Gamma \equiv \Gamma_{1}$ of our model. It only remains to find out the coupling $V$ and the group velocity $v$.

In order to quantify the coupling between the junction and the microwave guide we will need to explicitely make the calculations leading to the previous results and find out the harmonic approximations for the lowest energy levels. We begin by noticing that the energy minima are reached at a value of the phase

$$
\sin \left(\theta_{m}\right)=\frac{I}{I_{0}}=: r
$$

which is very close to $\pi / 2$, as predicted. Around this minimum, $\theta=\theta_{m}+\tilde{\theta}$, a harmonic approximation gives

$$
H \simeq E_{C} \hat{N}^{2}+\frac{1}{2} U^{\prime \prime} \tilde{\theta}^{2}
$$

with a curvature of the potential

$$
U^{\prime \prime}\left(\theta_{m}\right)=I_{0} \varphi_{0} \cos \left(\theta_{m}\right)=I_{0} \varphi_{0} \sqrt{1-r^{2}} .
$$

To diagonalize this Hamiltonian we introduce operators

$$
\begin{aligned}
& \hat{N}=\frac{1}{\sqrt{2} \alpha} i\left(a^{\dagger}-a\right), \\
& \tilde{\theta}=\frac{\alpha}{\sqrt{2}}\left(a+a^{\dagger}\right),
\end{aligned}
$$

with usual commutation relations, $\left[a, a^{\dagger}\right]=1$, and impose

$$
\frac{E_{C}}{2 \alpha^{2}}=\frac{U^{\prime \prime} \alpha^{2}}{4}=\frac{1}{2} \hbar \omega_{p} .
$$

This provides us with the plasma frequency,

$$
\omega_{p}=\sqrt{\frac{E_{C} U^{\prime \prime}}{2 \hbar^{2}}}=\sqrt{\frac{I_{0}}{4 \varphi_{0} C_{J}}}\left(1-r^{2}\right)^{1 / 4},
$$

but also with the "strength" of the number and phase operators,

$$
\alpha^{2}=\sqrt{\frac{2 E_{C}}{U^{\prime \prime}}}=\frac{E_{C}}{\hbar \omega_{p}},
$$

which is related to the charging energy and the plasma frequency in a simple way. 


\subsection{Qubit-line in the dipole approximation}

Given that the total capacitance of the transmission line is much larger than that of a single superconducting qubit, we can apply the dipole limit to study the coupling between both elements and assume that the interaction term goes as

$$
H=\hat{q} \frac{C_{g}}{C_{g}+C_{J}} \hat{V}(x, t) .
$$

Following the theory in Sec. 4, the potential created inside the waveguide can be written as a function of the charge distribution on the conductor

$$
\hat{V}(x, t)=\frac{1}{c} \frac{\partial \hat{Q}(x, t)}{\partial x} .
$$

Using the eigenvalue equation $\partial_{x} w_{k}(x, t)=i k w_{k}(x, t)$ and the dispertion relation (89), we obtain

$$
\hat{V}(x, t)=i \sum_{k} \sqrt{\frac{\hbar \omega_{k}}{2 c}}\left[a_{k} w_{k}(x, t)-a_{k}^{\dagger} w_{k}(x, t)^{*}\right] .
$$

If we assume that the incident and outgoing wavepackets have a small bandwidth, $\omega_{k} \simeq \omega_{0}$, we obtain the potential as a function of the propagating fields

$$
\hat{V} \simeq i \sqrt{\frac{\hbar \omega_{0}}{2 c}}\left[\psi_{r}+\psi_{l}-\psi_{r}^{\dagger}-\psi_{l}^{\dagger}\right]
$$

As a consistency check, we can consider the case of a small transmission line, forming a "cavity" or resonantor in which the modes are very well separated. In that limit we only need to consider a single momentum, $p$, and everything reduces to the formula

$$
\hat{V}(x, 0)=i \sqrt{\frac{\hbar \omega_{p}}{2 C}}\left(a_{p}-a_{p}^{\dagger}\right) .
$$

Here, $C=L \times c$ is the total capacity of the transmission line and the quantity $\left(\hbar \omega_{k} / 2 C\right)^{1 / 2}$ is the r.m.s. voltage. Note that, consistently with the expression in the experiment of Wallraff et al. [18, 19], the coupling between the qubit and a particular mode is inversely proportional to the square root of the microwave guide length, $L$. However, the coupling constant with the fields in Eq. (82) is insensitive to the total size of the transmission line.

\subsection{Final parameterization of the setup}

Following the previous considerations we will write the interaction Hamiltonian between

the junction and the microwave field as in Eq. (79) using the charge operator (74). The coupling between the stripline and the two lowest levels of the CBJJ is proportional to the constant

$$
V=\frac{C_{g}}{C_{J}+C_{g}} \frac{e}{\alpha} \sqrt{\frac{\hbar \omega_{0}}{c}} .
$$

Let us remind that $\omega_{0}$ is the photon frequency, $C_{g}$ is the gate capacitance between the junction and the waveguide, $c$ is the capacitance per unit length of the waveguide and $\alpha$ is the harmonic oscillator wavepacket size (78). 
In addition to the coupling strength we need to compute the group velocity $v$. The precise derivation is left for Sec. 4, but we advance that this can be written using properties of the waveguide

$$
v=\frac{1}{\sqrt{c l}}=\frac{1}{c Z_{0}}
$$

where $l$ is the inductance per unit length and $Z_{0}$ the impedance. When introduced in our scattering problem, this results in the effective decay rate $\gamma$

$$
\gamma=\frac{\hbar v}{V^{2}} \hbar \Gamma=\frac{\hbar / c Z_{0}}{c_{12}^{2} e^{2} \hbar \omega_{0} / \alpha^{2} c} \hbar \Gamma=\frac{\alpha^{2}}{c_{12}^{2}} \frac{\hbar}{e^{2} Z_{0}} \frac{\Gamma}{\omega_{0}},
$$

expressed in terms of $c_{12}=C_{g} /\left(C_{g}+C_{J}\right)$ for resonant qubits (i.e. no detuning). We want to remark that there are plenty of parameters to tune, so that realistic conditions of high efficiency are achievable.

In practice one would begin by fixing the number of superconducting absorbers that can be used, $N$. This fixes a value of $\gamma$ for which absorption is optimal and which determines the optimal decay rate of the first energy level

$$
\Gamma=\frac{c_{12}^{2}}{\alpha^{2}} \frac{Z_{0} e^{2}}{h} \omega_{0}
$$

As an example, let us compute the optimal configuration for one qubit. Using the numbers in Berkley's experiment [15], we will fix the qubit capacitance $C_{J}=4.8 \mathrm{pF}$ and $c_{12}=0.13$. The charging energy is about $E_{C} / \hbar=0.10 \mathrm{GHz}$, which together with a plasma frequency $\omega=5 \mathrm{GHz}$ gives $\alpha^{2}=0.02$. Putting the numbers together

$$
\Gamma=\frac{Z_{0}}{5144 \Omega} \omega_{0}
$$

so that for inductances from $10 \Omega$ to $100 \Omega$ this gives $\Gamma=10-100 \mathrm{MHz}$. Higher decay rates can be achieved by increasing $C_{g}$ or decreasing the charging energy. For instance, a factor 2 increase of $C_{g}$ gives a factor 4 increase in $\Gamma$ which now ranges from $30-300 \mathrm{MHz}$.

\section{Photons in transmission lines}

In this section we study the mathematical models and physical properties of a coaxial coplanar microwave transmission line. This device consists on a central conductor carrying the wave and enclosed by two conductors set to the ground plane. A simple but effective model for such a line is obtained by coupling inductances and capacitors as shown in Fig. 9, As we will show later on, if we denote by $l$ and $c$ the inductances and capacitances per unit length, this model predicts the propagation of microwave fields in the transmission line according to a dispersion relation

$$
\omega_{k}=v|k|=\frac{1}{\sqrt{c l}}|k|,
$$

where $\hbar k$ is the momentum of the photon, $\omega_{k}$ is the frequency and $v$ is the group velocity. 


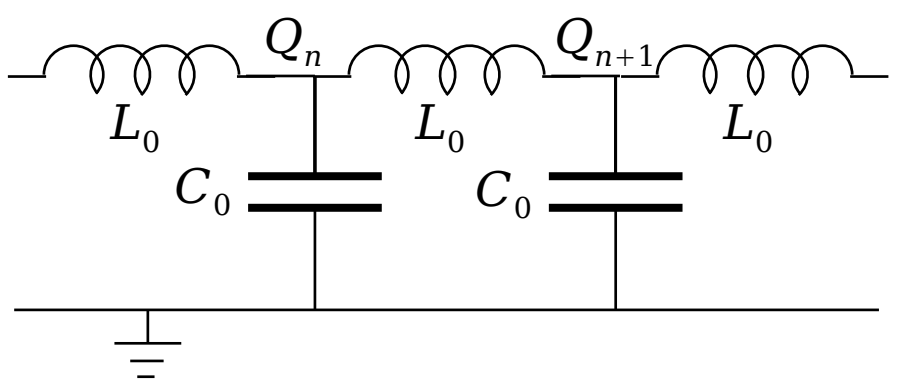

Figure 9. A transmission line can be modeled as a set of inductances and capacitances.

\subsection{Discrete model}

In order to analyze this circuit from Fig. 9, we must write down the Kirchhoff's law for the n-th block containing 4 nodes. When combining all equations and leaving as only variables the branch intensities, we obtain the set of second order differential equations

$$
-L_{0} \frac{d^{2} I_{n}}{d t^{2}}=\frac{1}{C_{0}}\left[2 I_{n}-I_{n-1}-I_{n+1}\right]
$$

These equations are similar to those describing an infinite set of oscillators of mass $m \propto L_{0}$ and spring constant $\kappa \propto 1 / C_{0}$. In analogy with the mechanical case, if we assume periodic boundary conditions to better reproduce propagation of charge, we find travelling wave solutions

$$
I_{n}(t)=I_{0} e^{i\left(k x_{n}-\omega t\right)}, k=\frac{2 \pi}{L} \times \mathbf{Z},
$$

where $x_{n}=a \times n, a$ is a parameter denoting the distance between neighbor oscillators,

$p$ is the momentum of the wave and $L$ is the length of the line. A direct substitution of this expression in Eq. (90), gives the dispertion relation

$$
\omega(k)=\left[\frac{2}{L_{0} C_{0}}(1-\cos (k a))\right]^{\frac{1}{2}} \simeq \sqrt{\frac{a^{2}}{L_{0} C_{0}}}|k|,
$$

which is approximately linear for small momenta, long waveguides or thin discretization. Using the inductance and capacitance per unit length

$$
l=L_{0} / a, c=C_{0} / a,
$$

we obtain the group velocity $v$ and dispersion relations introduced before (89).

\subsection{Lagrangian formalism and continuum limit}

The previous evolution equations (90) can be obtained from the Lagrangian

$$
L=\sum_{n}\left(\frac{L_{0}}{2} \dot{Q}_{n}^{2}-\frac{1}{2 C_{0}}\left(Q_{n}-Q_{n+1}\right)^{2}\right)
$$

using the Euler-Lagrange equations

$$
\frac{d}{d t}\left(\frac{\partial L}{\partial \dot{Q}_{n}}\right)=\frac{\partial L}{\partial Q_{n}}
$$


A more realistic model for a continous transmission line is obtained by taking the limit of infinitesimally small capacitors and inductors, $a \rightarrow 0$, while preserving the intensive values $l$ and $c$. In the continuum limit we replace the discrete charges by a continuous charge density distribution, $Q_{n} \simeq a \times q\left(x_{n}, t\right)$, which should be a smooth and integrable function of the position variable $x$. Through this procedure one obtains a total Lagrangian expressed in integral form

$$
L=\int d x\left[\frac{l}{2}\left(\partial_{t} q\right)^{2}-\frac{1}{2 c}\left(\partial_{x} q\right)^{2}\right] .
$$

The charge distribution described through the field $q(x, t)$ obeys a wave equation

$$
\partial_{x}^{2} q(x, t)-\frac{1}{v^{2}} \partial_{t}^{2} q(x, t)=0,
$$

where the group velocity is precisely the one already found in the discrete case.

\subsection{Hamiltonian and quantization}

The quantization of an electrical circuit is a three-step process. We begin by identifying the classical canonically conjugate variables: the charge distribution $q(x, t)$ and the associated momentum

$$
\Pi_{q}=\frac{\partial \mathcal{L}}{\partial \dot{q}}=l \dot{q}(x, t) .
$$

The Hamiltonian is then built using the prescription

$$
H=\int d x \Pi_{q} \dot{q}-L=\int d x\left[\frac{\left(\Pi_{q}\right)^{2}}{2 l}+\frac{\left(\partial_{x} q\right)^{2}}{2 c}\right] .
$$

Finally, the variables $\left\{q, \Pi_{q}\right\}$, are replaced with Hermitian operators $\left\{\hat{q}, \hat{\Pi}_{q}\right\}$, with commutation relations

$$
\left[\hat{q}(x, t), \hat{\Pi}_{q}\left(x^{\prime}, t\right)\right]=i \hbar \delta\left(x-x^{\prime}\right) .
$$

The previous Hamiltonian is quadratic and it can be diagonalized. We begin by analyzing the dynamics of these operators in the Heisenberg picture and noticing that the charge satisfies a wave equation (97). It therefore makes sense to look for a set of normal modes made of plane waves

$$
\begin{aligned}
& \hat{q}(x, t)=\sum_{k} N_{k} \hat{q}_{k}(t) u_{k}(x), \\
& u_{k}(x, t)=\frac{1}{\sqrt{\mathcal{L}}} \exp (i k x), k \in \frac{2 \pi}{\mathcal{L}} \times \mathbf{Z},
\end{aligned}
$$

where $\hat{q}_{k}$ are our new dynamical variables and $N_{k}$ is a normalization constant which will be fixed later on. Note that we are using periodic boundary conditions because they are best suited for describing transport, but we have not fixed the transmission line length $\mathcal{L}$, which may be arbitrarily large.

Replacing the expansion above in (97), produces second order differential equation for the unknown operators

$$
\ddot{\hat{q}}_{k}(t)+\omega_{k}^{2} \hat{q}_{k}(t)=0 .
$$


Since both $\hat{q}$ and $\hat{q}_{k}$ are physical observables and Hermitian operators, the most general solution is $\hat{q}_{k}(t)=a_{k} e^{-i \omega_{k} t}+a_{k}^{\dagger} e^{i \omega_{k} t}$, expressed in terms of a time independent operator, $a_{k}$. The commutation relation (100) produces both bosonic commutation relations for the $a_{k}$

$$
\left[a_{k}, a_{k^{\prime}}^{\dagger}\right]=\delta_{k k^{\prime}},\left[a_{k}, a_{k^{\prime}}\right]=\left[a_{k}^{\dagger}, a_{k^{\prime}}^{\dagger}\right]=0,
$$

and $N_{k}^{2}=\hbar /\left(2 \omega_{k} l\right)$. Finally, with the orthonormalized wave functions $w_{k}(x, t)=$ $u_{k}(x) \exp \left(-i \omega_{k} t\right)$, we arrive at

$$
\begin{aligned}
& \hat{q}(x, t)=\sum_{k} \sqrt{\frac{\hbar}{2 \omega_{k} l}}\left[a_{k} w_{k}(x, t)+\text { H.c. }\right] \\
& \hat{\Pi}_{q}(x, t)=\sum_{k} i \sqrt{\frac{\hbar \omega_{k} l}{2}}\left[a_{k}^{\dagger} w_{k}(x, t)^{*}-\text { H.c. }\right] .
\end{aligned}
$$

Given the specific form of the canonical operators, we may obtain a particular dispersion relation $\omega_{k}$ that diagonalizes the Hamiltonian. Using the relations

$$
\begin{aligned}
& \int w_{k}(x, t) w_{k^{\prime}}(x, t) d x=\delta_{k+k^{\prime}} e^{-i\left(\omega_{k}+\omega_{k^{\prime}}\right) t}, \\
& \int w_{k}(x, t) w_{k^{\prime}}(x, t)^{*} d x=\delta_{k-k^{\prime}}
\end{aligned}
$$

and imposing

$$
\frac{\hbar \omega_{k} l}{2} \times \frac{1}{2 l}=\frac{\hbar}{2 \omega_{k} l} \times \frac{1}{2 c} \times k^{2},
$$

we will be able to cancel all terms proportional to $a_{k} a_{-k}$ and $a_{k}^{\dagger} a_{-k}^{\dagger}$, obtaining a set of uncoupled oscillators

$$
H=\sum_{k} \hbar \omega_{k}\left(a_{k}^{\dagger} a_{k}+\frac{1}{2}\right)
$$

where the dispersion relation is strictly the one introduced before in Eq. (89).

\subsection{Linearization}

In our work we focus on states that contain photons with momenta around $\left|k_{0}\right|$ or $\omega_{0} / v$, where $\omega_{0}$ is the principal frequency of the wavepacket. We thus introduce two field operators representing the right- and leftward propagating photons,

$$
\begin{aligned}
\psi_{r}(x, t) & =\sum_{k \in \mathcal{B}} a_{k} w_{k}(x, t), \\
\psi_{l}(x, t) & =\sum_{k \in \mathcal{B}} a_{-k} w_{-k}(x, t),
\end{aligned}
$$

where $\mathcal{B}=\left[k_{0}-\Delta, k_{0}+\Delta\right]$ is the desired neighborhood around the principal momentum, characterized by a sensible cut-off $\Delta$. These two fields satisfy the evolution equations

$$
\begin{aligned}
& i \partial_{t} \psi_{r}(x, t)=-i \hbar v \partial_{x} \psi_{r}(x, t), \\
& i \partial_{t} \psi_{l}(x, t)=+i \hbar v \partial_{x} \psi_{l}(x, t),
\end{aligned}
$$


and we can write the effective Hamiltonian

$$
H=\int\left[\psi_{r}^{\dagger}\left(-i \hbar v \partial_{x}\right) \psi_{r}+\psi_{l}^{\dagger}\left(+i \hbar v \partial_{x}\right) \psi_{l}\right] d x .
$$

There is a catch in the previous expansion: the operators $\psi_{r, l}$ do not have the usual bosonic commutation relations. For instance

$$
\left[\psi_{r}(x, t), \psi_{r}^{\dagger}(y, t)\right]=\sum_{k \in \mathcal{B}} w_{k}(x, t) \bar{w}_{k}(y, t),
$$

where the right hand side represents a truncation of the distribution $\delta(x-y)$ within the interval of momental $\mathcal{B}$. In this work we are interested in the asymptotics of the absorption process, treating long times and long enough wavepackets for which the rotating wave approximation is valid. In this case we are justified to approximate

$$
\left[\psi_{\alpha}(x, t), \psi_{\beta}^{\dagger}(y, t)\right] \simeq \delta_{\alpha, \beta} \delta(x-y), \quad \alpha, \beta \in\{r, l\},
$$

and treat the right and left propagating fields as causal.

\section{Conclusions}

We have developed the theory of a possible microwave photon detector in circuit QED, and studied diverse regimes, advantages and difficulties with a realistic scope. Though we believe that our contribution will boost the theoretical interest and possible implementations in microwave photodetecion, we will summarize the potential limitations and imperfections of our proposal. First, the bandwidth of the detected photons has to be small compared to the time required to absorb a photon, roughly proportional to $1 / \Gamma$. Second, the efficiency might be limited by errors in the discrimination of the state $|g\rangle$ but these effects are currently negligible [29]. Third, dark counts due to the decay of the state $|0\rangle$ can be corrected by calibrating $\Gamma_{0}$ and postprocessing the measurement statistics. Fourth, fluctuations in the relative energies of states $|0\rangle$ and $|1\rangle$, also called dephasing, are mathematically equivalent to an enlargement of the incoming signal bandwidth by a few megahertz and should be taken into account in the choice of parameters. Finally, and most important, unknown many-body effects cause the non-radiative decay process $1 \rightarrow 0$, which may manifest in the loss of photons while they are being absorbed. In current experiments [29], this happens with a rate of a few megahertzs, so that it would only affect long wavepackets.

Our design can be naturally extended to implement a photon counter using a number of detectors large enough to capture all incoming photons. Furthermore, our proposal can be generalized to other level schemes and quantum circuits that can absorb photons and irreversible decay into long lived and easily detectable states.

We expect to have contributed to the emerging field of detection of travelling photons. Its success may open the doors to the arrival of "all-optical" quantum information processing with propagating quantum microwaves. 


\section{Acknowledgments}

The authors thank useful feedback from P. Bertet, P. Delsing, D. Esteve, M. Hofheinz, J. Martinis, G. Johansson, M. Mariantoni, V. Shumeiko, D. Vion, F. Wilhelm and C. Wilson. G.R. acknowledges financial support from CONICYT grants and PBCT-Red 21, and hospitality from Univ. del País Vasco and Univ. Complutense de Madrid. J.J.G.R. received support from Spanish Ramon y Cajal program, and projects FIS2006-04885

and CAM-UCM/910758. E.S. thanks support from Ikerbasque Foundation, UPV-EHU Grant GIU07/40, and EU project EuroSQIP.

\section{References}

[1] G. Romero, J. J. García-Ripoll, and E. Solano, Phys. Rev. Lett. 102, 173602 (2009).

[2] Y. Makhlin, G. Schön, and A. Shnirman, Rev. Mod. Phys. 73, 565 (2001).

[3] J. Q. You and F. Nori, Physics Today 58, 110000 (2005).

[4] R. J. Schoelkopf and S. M. Girvin, Nature 451, 664 (2008).

[5] J. Clarke and F. K. Wilhelm, Nature 453, 1031 (2008).

[6] V. Bouchiat, D. Vion, P. Joyez, D. Esteve, and M. H. Devoret, Phys. Scr. T76, 165 (1998).

[7] Y. Nakamura, Yu. A. Pashkin, and J. S. Tsai, Nature 398, 786 (1999).

[8] D. Vion, A. Aasime, A. Cottet, P. Joyez, H. Pothier, C. Urbina, D. Esteve, and M. H. Devoret, Science 296, 886 (2002).

[9] T. Yamamoto, Yu. A. Pashkin, O. Astafiev, Y. Nakamura, and J. S. Tsai, Nature 425, 941 (2003).

[10] J. E. Mooij, T. P. Orlando, L. Levitov, L. Tian, C. H. van der Wal, and S. Lloyd, Science 285, 1036 (1999).

[11] C. H. van de Wal, A. C. J. ter Haar, F. K. Wilhelm, R. N. Schouten, C. J. P. M. Harmans, T. P. Orlando, S. Lloyd, and J. E. Mooij, Science 290, 773 (2000).

[12] I. Chiorescu, Y. Nakamura, C. J. P. M. Harmans, and J. E. Mooij, Science 299, 1869 (2003).

[13] Frank Deppe, Matteo Mariantoni, E. P. Menzel, A. Marx, S. Saito, K. Kakuyanagi, H. Tanaka, T. Meno, K. Semba, H. Takayanagi, E. Solano, and R. Gross, Nature Physics 4, 686 (2008).

[14] J. M. Martinis, M. H. Devoret, and J. Clarke, Phys. Rev. Lett. 55, 1543 (1985).

[15] A. J. Berkley, H. Xu, R. C. Ramos, M. A. Gubrud, F. W. Strauch, P. R. Johnson, J. R. Anderson, A. J. Dragt, C. J. Lobb, and F. C. Wellstood, Science 300, 1548 (2003).

[16] R. W. Simmonds, K. M. Lang, D. A. Hite, S. Nam, D. P. Pappas, and J. M. Martinis, Phys. Rev. Lett. 93, 077003 (2004).

[17] M. Steffen, M. Ansmann, R. C. Bialczak, N. Katz, E. Lucero, R. McDermott, M. Neeley, E. M. Weig, A. N. Cleland, and J. M. Martinis, Science 313, 1423 (2006).

[18] A. Blais, R.-S. Huang, A. Wallraff, S. M. Girvin, and R. J. Schoelkopf, Phys. Rev. A 69, 062320 (2004).

[19] A. Wallraff, D. Schuster, A. Blais, L. Frunzio, R.-S. Huang, J. Majer, S. Kumar, S. M. Girvin, and R. J. Schoelkopf, Nature 43, 162 (2004).

[20] I. Chiorescu, P. Bertet, K. Semba, Y. Nakamura, C. J. P. M: Harmans, and J. E. Mooij, Nature 431, 159 (2004).

[21] D. Bouwmeester, A. Ekert, and A. Zeilinger, The Physics of Quantum Information (Springer Verlag, Berlin, 2008).

[22] Pieter Kok, W. J. Munro, Kae Nemoto, T. C. Ralph, Jonathan P. Dowling, and G. J. Milburn, Rev. Mod. Phys. 79, 135 (2007).

[23] U. Leonhardt, Measuring the Quantum State of Light (Cambridge University Press, Cambridge, 1997).

[24] D. Leibfried, R. Blatt, C. Monroe, and D. Wineland, Rev. Mod. Phys. 75, 281 (2003). 
[25] M. Mariantoni, M. J. Storcz, F. K. Wilhelm, W. D. Oliver, A. Emmert, A. Marx, R. Gross, H. Christ, and E. Solano, arXiv:cond-mat/0509737.

[26] R. Gross group at Walther-Meissner Institute (Garching, Germany), private communication.

[27] B. G. U. Englert, G. Mangano, M. Mariantoni, R. Gross, J. Siewert, and E. Solano, arXiv:0904.1769.

[28] J. Majer, J. M. Chow, J. M. Gambetta, J. Koch, B. R. Johnson, J. A. Schreier, L. Frunzio, D. I. Schuster, A. A. Houck, A. Wallraff, A. Blais, M. H. Devoret, S. M. Girvin, and R. J. Schoelkopf, Nature 449, 443 (2007).

[29] M. Hofheinz, E. M. Weig, M. Ansmann, C. B. Radoslaw, E. Lucero, M. Neeley, A. D. O'Connell, H. Wang, J. M. Martinis, and A. N. Cleland, Nature 454, 310 (2008).

[30] M. Hofheinz, H. Wang, M. Ansmann, Radoslaw C. Bialczak, Erik Lucero, M. Neeley, A. D. O'Connell, D. Sank, J. Wenner, John M. Martinis, and A. N. Cleland, Nature 459, 546 (2009).

[31] D. I. Schuster, A. A. Houck, J. A. Schreier, A. Wallraff, J. M. Gambetta, A. Blais, L. Frunzio, J. Majer, B. Johnson, M. H. Devoret, S. M. Girvin, and R. J. Schoelkopf, Nature 445, 515 (2007).

[32] A. A. Houck, D. I. Schuster, J. M. Gambetta, J. A. Schreier, B. R. Johnson, J. M. Chow, L. Frunzio, J. Majer, M. H. Devoret, S. M. Girvin, and R. J. Schoelkopf, Nature 449, 328 (2007).

[33] F. Helmer, M. Mariantoni, E. Solano, and F. Marquardt, Phys. Rev. A 79, 052115 (2009).

[34] Shwetank Kumar and David P. DiVincenzo, arXiv:0906.2979.

[35] J. M. Fink, M. Göppl, M. Baur, R. Bianchetti, P. J. Leek, A. Blais, and A. Wallraff, Nature 454, 315 (2008).

[36] L. S. Bishop, J. M. Chow, J. Koch, A. A. Houck, M. H. Devoret, E. Thuneberg, S. M. Girvin, and R. J. Schoelkopf, Nature Physics 5, 105 (2009).

[37] Matteo Mariantoni, Frank Deppe, A. Marx, R. Gross, F. K. Wilhelm, and E. Solano, Phys. Rev. B 78, 104508 (2008).

[38] F. Helmer, M. Mariantoni, A. G. Fowler, J. von Delft, E. Solano, and F. Marquardt, Europhys. Lett. 85, 50007 (2009).

[39] Lan Zhou, Z. R. Gong, Yu-xi Liu, C. P. Sun, and Franco Nori, Phys. Rev. Lett. 101, 100501 (2008).

[40] J.-T. Shen and S. Fan, Phys. Rev. Lett. 95, 213001 (2005).

[41] P. K. Day, H. G. LeDuc, B. A. Mazin, A. Vayonakis, and J. A. Zmuidzinas, Nature 425, 817 (2003).

[42] M. H. Devoret, A. Wallraff, and J. M. Martinis, arXiv:cond-mat/0411174. 